\title{
Human Ciliopathies: From Ponds And Tidal Pools, To The Laboratory, To The Bedside
}

\author{
J.L. Carson,* M. Zariwala**, M. R. Knowles***
}

*Department of Pediatrics and Center for Environmental Medicine, Asthma, and Lung Biology, **Department of Pathology and Laboratory Medicine, ***Department of Medicine, The University of North Carolina at Chapel Hill, Chapel Hill, NC 27599

In the early years of the twentieth century a German physician, A. K. Siewert, published a manuscript on the occurrence of situs inversus among his patients with bronchiectasis. This may have been not only the first case report of primary ciliary dyskinesia (PCD) but also one of the first reports of the clinical manifestations of a heritable disease. During the 1970s, the advent of routine biological electron microscopy led directly to the discovery of the pathophysiologic basis for the syndrome and its presentation; that is, structural defects in ciliary axonemes. Because patients with PCD typically presented with the disparate symptoms of respiratory disease, infertility, and situs issues, it was assumed that the syndrome was inherited and that affected organ systems were impacted in some fashion by abnormal ciliary/flagellar function. Recognizing the highly conserved nature of ciliary and flagellar proteins, researchers late in the twentieth century recognized the potential of the eukaryotic Protist Chlamydomonas as well as other invertebrates as potentially useful molecular genetic tools for the identification of candidate genes for PCD. These investigations have led in recent years to the discovery of several loss-of-function mutations that cause PCD. Thus it was that not only was PCD likely one of the first reported diseases of genetic origin but also the first of a new class of human diseases known as ciliopathies. The ciliopathies include a variety of seemingly disparate clinical presentations that include infertility, acute and chronic respiratory disease, heart disease and laterality defects, hearing loss, kidney disease, and obesity syndromes; but all have as their pathophysiologic basis structure/function abnormalities of cilia.

Tracking the pathophysiologic basis for the role of cilia in human disease almost certainly had its origins in the ability to observe the cellular characteristic for which cilia are best known, their role as an organelle of motility. However, in recent years, considerable attention has been drawn to the role of the cilium as a sensory organelle. Indeed, the function of motility may have emerged secondarily from the initial sensory functions of the cilium. Unlike the motile cilia that line mucosal surfaces and serve the motility function of male gametes, primary cilia have no central microtubular pair and exhibit either no motility or alternatively a clockwise whirling motility. Once considered a vestigial organelle, primary cilia now have well characterized functions in the detection of mechanical and chemical signals and signal transduction and in the control of cell growth. Of particular significance in human disease, primary cilia are thought to be involved in establishment of appropriate visceral positioning and failure of this mechanism may be responsible for conferring laterality disorders. Further, polycystic kidney disease, hepatic and pancreatic abnormalities, and certain sensory defects appear to be associated with deficits in intraflagellar transport (IFT), a mechanism for ciliary assembly, maintenance, and signaling.

Our understanding of the molecular genetics of ciliary abnormalities has advanced considerably in the last decade, however there remains another component of the ciliopathies that is associated with 
infection, irritant exposure, and inflammation. These anomalies are typically considered secondary and transient and exhibit a different spectrum of structural compromise relative to the primary genetic anomalies. Nevertheless, virtually every human is subject at some point to morbidity from the effects of failed ciliary function deriving from epithelial injury. Moreover, individuals with congenital ciliary diseases, particularly PCD, may have secondary ciliary dysfunction imposed by more frequent episodes of infection.

Microscopic imaging technologies have played a historic role in the discovery of ciliopathies and the elucidation of their pathophysiology. As advances are made in achieving a comprehensive understanding of their genetic basis, both existing and emerging imaging technologies will continue to play a pivotal role in research, diagnosis, and the development of new and improved approaches to therapy.

This work was supported by the Primary Ciliary Dyskinesia Foundation, a Clinical Innovator Award from the Flight Attendant Medical Research Institute (JLC), and National Institutes of Health Grant U54RR019480. 